\title{
Adaptive robust energy and reserve co-optimization of integrated electricity and heating system considering wind uncertainty
}

Tan, Jin; Wu, Qiuwei; Hu, Qinran; Wei, Wei; Liu, Feng

Published in:

Applied Energy

Link to article, DOI:

10.1016/j.apenergy.2019.114230

Publication date:

2020

Document Version

Peer reviewed version

Link back to DTU Orbit

Citation (APA):

Tan, J., Wu, Q., Hu, Q., Wei, W., \& Liu, F. (2020). Adaptive robust energy and reserve co-optimization of integrated electricity and heating system considering wind uncertainty. Applied Energy, 260, [114230]. https://doi.org/10.1016/j.apenergy.2019.114230

\section{General rights}

Copyright and moral rights for the publications made accessible in the public portal are retained by the authors and/or other copyright owners and it is a condition of accessing publications that users recognise and abide by the legal requirements associated with these rights.

- Users may download and print one copy of any publication from the public portal for the purpose of private study or research.

- You may not further distribute the material or use it for any profit-making activity or commercial gain

- You may freely distribute the URL identifying the publication in the public portal 


\title{
Adaptive robust energy and reserve co-optimization of integrated electric-
} ity and heating system considering wind uncertainty

\author{
Jin Tan ${ }^{\mathrm{a}}$, Qiuwei $\mathrm{Wu}^{\mathrm{a}, *}$, Qinran $\mathrm{Hu}^{\mathrm{b}}$, Wei Wei ${ }^{\mathrm{c}}$, Feng $\mathrm{Liu}^{\mathrm{c}}$ \\ ${ }^{a}$ Center for Electric Power and Energy, Technical University of Denmark, Kgs. Lyngby, 2800 Denmark \\ $b$ School of Electrical Engineering, Southeast University, 2 Sipailou, Nanjing, China \\ c Department of Electrical Engineering, Tsinghua University, Beijing, China, 100084
}

\begin{abstract}
The uncertainty and variability of wind power pose significant challenges to secure and reliable operation of power systems. Coordinated operation of the electric power system and district heating system, which can provide sufficient reserve capacity and flexibility, is an effective way to cope with the uncertainty. This paper proposes an adaptive robust energy and reserve co-optimization for the integrated electricity and heat system to minimize the total system cost under the worst-case realization of wind uncertainty considering spatial correlations of wind uncertainties. The available reserve capacity and flexibility provided by the district heating system is fully used by exploiting the regulation capabilities of combined heat and power units and electrical boilers, as well as utilizing the building thermal inertia. To reduce the conservatism of the robust solution, the spatial correlation of wind uncertainties is considered in the uncertainty set. The column-and-constraint generation algorithm is adopted to solve the adaptive robust model iteratively by reformulating the second stage with its Karush-Kuhn-Tucker conditions. Simulation results demonstrate that the economic efficiency is improved by utilizing the reserve flexibility from the district heating system and considering wind farm spatial correlations. Compared with the conventional single-stage optimized model, the feasibility of the two-stage robust solution is always guaranteed by considering the real-time operation constraints of the electric power system and district heating system.
\end{abstract}

\section{Highlights}

A two-stage adaptive robust energy and reserve co-optimization scheme is proposed.

The spatial correlation of wind uncertainty is used to control the conservatism of robust solutions.

The available reserve capacity of combined heating and power units is fully utilized.

Electrical boiler and buildings' thermal inertia are introduced to increase the reserve capacity.

\section{Keywords}

Adaptive robust optimization, energy and reserve co-optimization, integrated electricity and heating system, wind power uncertainty.

\begin{tabular}{l} 
Indices and sets \\
$\phi^{N / G / W D}$ \\
$\phi^{C H P / E B}$ \\
$\phi^{N D / H S / H D}$ \\
$\phi^{S / R, p i p e}$ \\
$\Omega_{n}^{C H P / E B}$ \\
\hline
\end{tabular}

Set of buses/thermal units/wind farms in EPS

Set of CHP units/EBs

Set of heat nodes/heat sources/heat loads in DHS

Set of supply pipelines/return pipelines in DHS

Set of CHP units/electrical boilers connected to bus $n$ 


\begin{tabular}{|c|c|}
\hline$\Omega_{n}^{G / W D / L D / N}$ & Set of thermal units/wind farms/electric loads/buses connected to bus $n$ \\
\hline$\Omega_{j}^{H S / C H P / E B}$ & Set of heat nodes/ CHP units/EBs connected to heat source $j$ \\
\hline$\Omega_{n d}^{H S / H L}$ & Set of heat sources/ heat loads connected to heat node $n d$ \\
\hline$\Omega_{n d}^{S / R, p i p e}$ & Set of supply pipelines/return pipelines connected to heat node $n d$ \\
\hline$T$ & Set of hours \\
\hline$D A / R T / H S / H D$ & Day-ahead stage/Real-time stage/heat source/heat load \\
\hline \multicolumn{2}{|l|}{ Variables } \\
\hline$P_{h, t}^{C H P}, P_{g, t}^{G}$ & Electricity output of CHP/non-CHP thermal unit \\
\hline$P_{i, t}^{E B}$ & Electricity consumption of electric boiler \\
\hline$R_{h, t}^{C H P, U / D}, R_{g, t}^{G, U / D}$ & Upward/downward reserve capacity of CHP/non-CHP thermal unit \\
\hline$r_{h, t}^{C H P, U / D}, r_{g}^{G, U / D}$ & Upward/downward regulation of CHP/non-CHP thermal unit \\
\hline$L_{l, t}^{\text {shed }}$ & Load shedding \\
\hline$\Delta W_{w, t}$ & Deviation of wind power \\
\hline$W_{w, t}^{\text {spill }}$ & Wind spillage \\
\hline$\delta_{n, t}$ & Phase angle of buses \\
\hline$H_{h, t}^{C H P}, H_{i, t}^{E B}$ & Heat output of CHP unit/electric boiler \\
\hline$\tau_{n d, t}^{S / R}$ & Temperature of node $n d$ in the supply/return network \\
\hline$\tau_{p, t}^{S / R, \text { in }}, \tau_{p, t}^{S / R, \text { out }}$ & Mass flow temperature at the inlet/outlet of pipeline $p$ in the supply/return network \\
\hline$H_{l, t}^{H D}$ & Heat supply at heat load aggregator \\
\hline$H_{l, t}^{\text {Build }}$ & Actual heat consumption of buildings at heat load aggregator \\
\hline$T_{l, t}^{i n}$ & Indoor temperature of buildings at heat load aggregator \\
\hline \multicolumn{2}{|l|}{ Parameters } \\
\hline$C_{h}^{C H P}$ & Energy offer price of CHP units \\
\hline$C_{g}^{G}$ & Energy offer price of non-CHP thermal units \\
\hline$C_{h}^{+/-}, C_{g}^{+/-}$ & Upward/downward reserve offer price of CHP units/non-CHP thermal units \\
\hline$C_{l}^{\text {shed }}$ & Cost coefficient for lost load \\
\hline$B_{n m}$ & Susceptance of transmission line $\mathrm{nm}$ \\
\hline$P_{w, t}^{w e}$ & Predicted wind power \\
\hline$P_{l, t}^{\text {load }}$ & Electric load \\
\hline$P R_{h, t}^{C H P, \text { up } / \text { down }}, P R_{g, t}^{G, \text { up } / \text { down }}$ & Upward/downward ramping rate limit of CHP/non-CHP thermal unit \\
\hline$P_{h, t}^{C H P, \max }, P_{g, t}^{G, \max }, P_{i, t}^{E B, \max }, \Delta W_{w, t}^{\max }$ & $\begin{array}{l}\text { Maximum value of CHP output/thermal unit output/power consumption of electric } \\
\text { boiler/wind power deviation }\end{array}$ \\
\hline$P_{h, t}^{C H P, \min }, P_{g, t}^{G, \min }$ & Minimum value of CHP output/thermal unit output \\
\hline$\eta_{h}^{C H P}, \eta_{i}^{E B}$ & Heat to power coefficient of $\mathrm{CHP} /$ power to heat coefficient of electric boiler \\
\hline$c, l_{p}$ & Specific heat capacity of water/length of pipelines \\
\hline$m_{j, t}^{H S}, m_{l, t}^{H D}, m_{p, t}^{S / R, p i p e}$ & Mass flow rate of heat sources/heat loads/supply pipes/return pipes \\
\hline$\tau_{t}^{a}, T_{l, t}^{i n, \max / \min }$ & Ambient temperature of pipelines/maximum/minimum indoor temperature \\
\hline$c_{\text {air }}, \rho_{\text {air }}$ & Air specific heat/indoor air density \\
\hline
\end{tabular}




\section{Introduction}

Recently, the integrated energy system (IES) has become a promising paradigm of the future energy system. In the IES, different energy vectors are connected with each other through coupling components [1]. Combined heat and power (CHP) units, which produce electricity and heat simultaneously, are widely used in the countries and regions with cold winters due to its high efficiency, e.g., Denmark and Northern China [2]. In this regard, the electric power system (EPS) and district heating system (DHS) are interdependent with each other and the joint operation of the integrated electricity and heat system (IEHS) can improve energy efficiency and reduce $\mathrm{CO}_{2}$ emissions [3]. However, the power and heat output of CHP units are determined by heat demand, which limits further integration of wind power. In the winter, the peak demand of heat loads and the peak generation of wind power usually happen during night, while the electricity demands are quite low at this time [4]. To meet the heat demand, CHP units are dispatched and generate electricity proportionally, which leads to high wind power curtailment.

In order to better integrate wind power, many efforts have been done to enhance the flexibility of CHP units. The impact of thermal energy storage (TES) on the flexible operation of CHP was investigated in [5]. TES can transform surplus wind power to heat and store it in the heat tank, and discharge the heat to DHS when heat demand is high. However, the investment for energy storage devices is quite high. The benefits of electrical boilers (EBs) and heat pumps (HPs) in the DHS to relax the coupling between heat and power generation in CHP units, which could facilitate the integration of wind power, were studied in [6]-[7]. In [8], the time delay of the temperature change of district heating networks (DHNs) was studied and the heat storage capacity of DHNs was utilized to accommodate more wind power. Beside the measures from source and network sides, the demand side also has huge potential to improve the flexibility of DHS by taking into account the thermal dynamic characteristics and acceptable temperature tolerance of heat consumers [9]-[10]. In [11], the thermal dynamic model was integrated into the energy system for community grids and the energy management was achieved in a distributed way.

The studies above mainly focus on reducing the wind power spillage by improving the flexibility of energy schedules. However, the uncertainty and limited predictability of wind power also raise rigorous challenges to the secure and reliable operation of power systems [12]. As described in [13], the impacts of wind forecast errors on the real-time adjustments is significant, which is considered in the economic dispatch. Accordingly, an increased amount of reserve capacities is required to enable power plants to adjust their production in real time, and thus avoid contingencies caused by unexpected variations of wind power [14]. One conventional approach is that all of the reserve requirements are imposed on non-CHP thermal units [15]-[16]. However, this approach is often costly and quite conservative, which limits the operational flexibility of generators. Actually, with aforementioned measures to improve the flexibility of CHP units, the DHS can provide certain reserve capacity for the EPS [17]-[18]. The energy and reserve co-optimization considering the reserve capacity of CHP units has been discussed in [19]-[21]. A joint-dispatch model of energy and reserve for the CCHP-based MG was proposed in [19], which considers the dynamic process of cooling and heating systems. Ref. [20] proposed the centralized and decentralized co-optimization of power and reserves in transmission and distribution power markets based on both cyber and physical systems. However, the heat balance and physical operation constraints of CHP microgenerators are not taken into account. An optimal dispatch strategy for the IES was proposed in [21], which integrates the natural gas system and its security constraints, as well as using CHP units' reserve capacity.

In the aforementioned studies, CHP units are assumed to provide full reserve capacity as thermal units without accounting for real-time operation constraints of DHS. As such, the real-time regulation might be infeasible after the reserve optimized in the dayahead stage is deployed [22]. Therefore, the two-stage optimization framework is constructed to ensure the feasibility of the firststage decisions with the consideration of operation constraints in the second stage. Ref. [23] proposed a two-stage stochastic optimization model for the unit commitment under gas-supply uncertainty, where the real-time operation is considered. In [24], the 
day-ahead energy and reserve are determined using two-stage robust optimization under the worst-case realization of wind uncertainty, where the obtained solutions are feasible for all uncertain scenarios. Moreover, another solution to properly utilize the feasible reserve capacity and regulation of CHP units is to build a proper regulation model. Ref. [4] proposed a feasible region model for the DHS taking into account the building thermal inertia, which can be directly used in the central dispatch. A regulating region method to describe the heating-restricted reserve capacity of CHP units was proposed in [25].

However, the reserve capacity schedule for the IEHS in the studies mentioned above are optimized with minimum reserve requirements or allocated based on a proportionate amount. As such, the schedule problem boils down to a deterministic optimization and the optimality of the final solution is not guaranteed [26]. With the increasing penetration of wind power, it is essential to determine the reserve allocations adaptive to wind uncertainty. Ref. [27] presented a fuzzy-based method for the energy and reserve optimization. However, it is difficult for system operators to choose proper fuzzy membership functions representing the bound of uncertainty sets. In [28], stochastic optimization (SO) was adopted to optimize the energy and reserve dispatches jointly based on a number of scenarios representing the wind uncertainty, but the computational burden is heavy in order to ensure the performance of solutions. The robust optimization (RO), without requiring distribution information of uncertain parameters, has been extensively studied in the optimal operation of power systems, e.g., security constrained unit commitment (SCUC) [29]-[30] and security constrained economic dispatch (SCED) [31]. However, the RO has not been well studied to investigate the energy and reserve co-optimization for the IEHS, where the DHS is able to provide reserve capacity.

Therefore, in order to ensure the economic and secure operation for the IEHS, this paper proposes a two-stage robust energy and reserve co-optimization scheme to improve the economic efficiency while maintaining the operation security under the worst realization of wind power by utilizing the feasible reserve capacity provided by the DHS. The major contributions of this paper are as follows.

(i) The energy and reserve schedule for the IEHS is formulated as a two-stage optimization problem. Compared to the existing optimization framework, the physical operation constraints for the real-time corrective adjustments of the EPS and DHS are considered to ensure the feasibility of day-ahead schedules.

(ii) An adaptive robust co-optimization scheme for the IEHS is proposed to maintain the secure operation against all possible realizations of wind power uncertainty. The spatial correlation of wind uncertainties is incorporated to control the conservatism of robust solutions.

(iii) The additional reserve capacity provided by the DHS is fully used by exploiting the regulation capabilities of CHP units and EBs, as well as utilizing building thermal inertia, which can compensate the wind forecast error with higher economic efficiency.

The rest of the paper is organized as follows. The structure of the IEHS and mathematical model of two-stage robust energy and reserve co-optimization for the IEHS are described in Section 2. Section 3 details the solution method for the adaptive robust optimization model. In Section 4, the case studies and simulation results are given and discussed. Finally, the conclusions are drawn in Section 5.

\section{Two-stage adaptive robust energy and reserve co-optimization for IEHS}

2.1 Overview of IEHS

The proposed IEHS consists of the EPS and DHS, which are connected by coupling components. The schematic structure of an IEHS is shown in Fig. 1. Electric loads are supplied by wind power, thermal units and CHP units, while heat loads are met by CHP units and EBs. Electric power and heat are provided to consumers through the EPS and DHS, respectively.

DHS have different operation modes, including constant mass flow and constant temperature (CM-CT), constant mass flow and variable temperature (CM-VT), variable mass flow and constant temperature (VM-CT), or variable mass flow and variable temperature (VM-VT) [32]. In this paper, it is assumed that the DHN is under the CM-VT control strategy, as the heat loss has been proved independent of mass flow rate [33]. As such, the model of DHS is linear. 


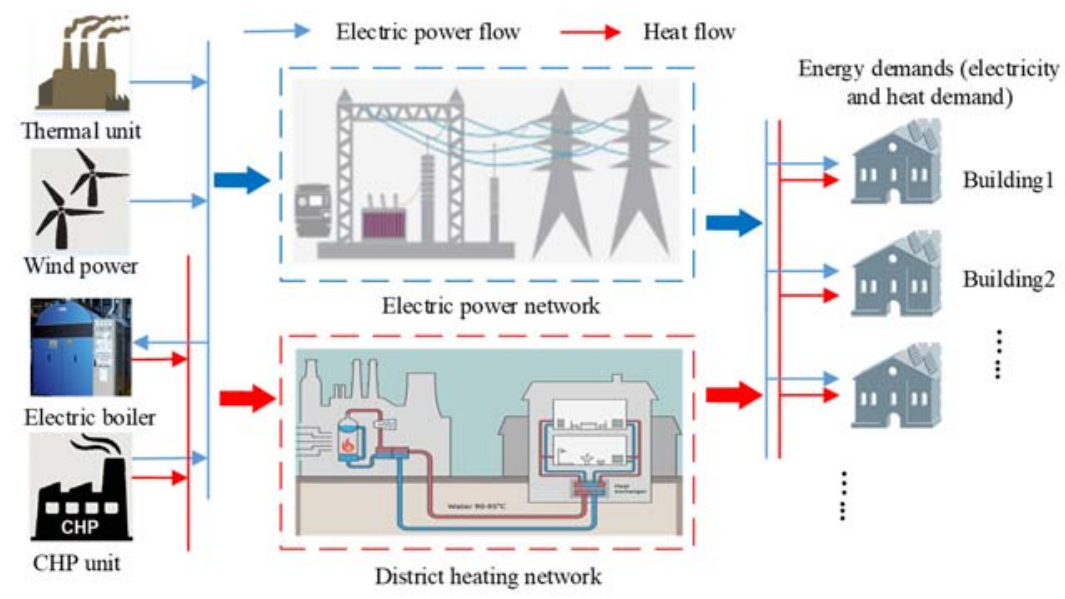

Fig. 1. Schematic structure of an integrated electricity and heating system

\subsection{Objective function}

A two-stage robust energy and reserve co-optimization scheme for the IEHS is formulated. The first-stage problem is to determine the day-ahead energy and reserve schedule with predicted wind power. The second-stage problem corresponds to the realtime regulations to address the error between actual wind power and the predicted one. An adaptive robust approach is adopted to formulate this type of two-stage optimization problem [34], considering the worst-case scenario of wind uncertainty. In this regard, the proposed scheme is cast as the following min-max-min form [35]. The objective is to minimize the total system cost of the IEHS in both day-ahead and real-time stages, as shown in (1). The max-min calculation in the objective function represents the regulation cost in the worst-case realization of wind power within an uncertainty set. In the proposed model, not only non-CHP units but also CHP units provide reserve capacity for the power system to cope with uncertainties. Moreover, the operation constraints of the EPS and DHS in the real-time operation are also considered. Thus, the feasibility of energy and reserve schedule is always ensured.

$$
\begin{aligned}
& \min \sum_{t \in T}\left(\sum_{h \in \Phi^{\mathrm{CHP}}} C_{h}^{G, C H P} P_{h, t}^{C H P}+C_{h}^{H, C H P} H_{h, t}^{C H P}+\sum_{g \in \Phi^{G}} C_{g}^{G} P_{g, t}^{G}+\sum_{h \in \Phi^{\mathrm{CHP}}}\left(C_{h}^{+} R_{h, t}^{C H P, U}+C_{h}^{-} R_{h, t}^{C H P, D}\right)+\sum_{g \in \Phi^{G}}\left(C_{g}^{+} R_{g, t}^{G, U}+C_{g}^{-} R_{g, t}^{G, D}\right)\right) \\
& +\max _{\Delta W_{w, t}} \min \sum_{t \in T}\left(\sum_{h \in \Phi^{\mathrm{CHP}}} C_{h}^{G, C H P}\left(r_{h, t}^{C H P, U}-r_{h, t}^{C H P, D}\right)+C_{h}^{H, C H P}\left(H_{h, t}^{C H P, R T}-H_{h, t}^{C H P}\right)+\sum_{g \in \Phi^{G}} C_{g}^{G}\left(r_{g, t}^{G, U}-r_{g, t}^{G, D}\right)+\sum_{l \in \Phi^{E L}} C_{l}^{\text {shed }} L_{l, t}^{\text {shed }}\right)
\end{aligned}
$$

\subsection{Mathematical formulation of EPS and DHS operations in two stages}

The day-ahead energy and reserve schedule is optimized in the first stage and will be kept in the second stage (real-time regulation). Both of day-ahead and real-time operation constraints of the IEHS consist of the constraints of EPS and DHS, respectively.

\subsubsection{Uncertainty set description}

In the RO, it is of great importance to construct a suitable uncertainty set. Since the predicted wind generation $P_{w, t}^{w e}$ adopted in the first stage is inaccurate, there is an error after the wind generation is revealed in the second stage. Generally, the wind uncertainty is represented by a box uncertainty set that is a polyhedron, which is described as (2). The whole uncertainty set includes all possible deviations from the predicted wind generation.

$$
P_{w, t}^{w e}-\Delta W_{w, t}^{\max } \leq \tilde{P}_{w, t} \leq P_{w, t}^{w e}+\Delta W_{w, t}^{\max }, \quad \forall w \in \Phi^{W D}, \forall t \in T
$$

Due to the geographically close location of wind farms and the inertia of local meteorological system, there is a spatial correlation between the stochastic outputs of neighboring wind farms. The correlation between wind farms is obtained through linear 
137 models based on the statistical data, which is expressed as $a_{q u, t} P_{u, t}^{w e}+b_{q u, t}$. According to [36], $\left.\widetilde{P_{q, t}}-\left(a_{q u, t} P_{u, t}^{w e}+b_{q u, t}\right)\right] / \sigma_{q u, t}$ fol138 lows the $t$-distribution, where $\sigma_{q u, t}$ is the standard deviation. Since the correlation between wind farms is also uncertain, the correlation is formulated in an interval with a certain confidence level $(2 \alpha-1)$, which is described as ,

$$
\left(a_{q u, t} P_{u, t}^{w e}+b_{q u, t}\right)-t_{n-2}^{\alpha} \sigma_{q u t} \leq \tilde{P}_{q, t} \leq\left(a_{q u, t} P_{u, t}^{w e}+b_{q u, t}\right)+t_{n-2}^{\alpha} \sigma_{q u, t}, \quad \forall q, u \in \Phi^{W D}, \forall t \in T
$$

Taking into account the spatial correlation of wind farms, the uncertainty set is described as (4). The solution of the proposed robust energy and reserve co-optimization scheme is less conservative by eliminating the extreme scenarios that unlikely happen.

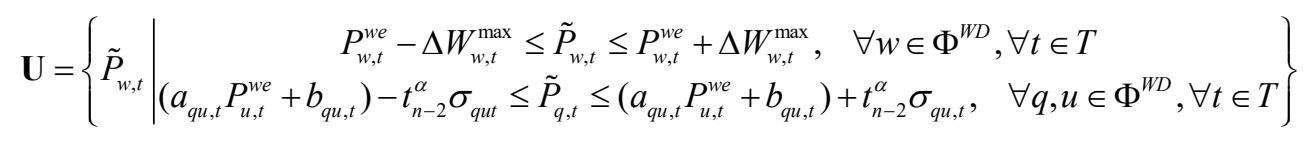

\subsubsection{Day-ahead operation constraints of EPS}

In this stage, the schedules of electric power and heat output are optimized and the reserve capacity is determined to cope with wind uncertainties. The DC power flow is employed in this optimization model. At each bus of the EPS, the total generation and electric loads must be balanced at all times:

$$
\sum_{h \in \Omega_{n}^{C H P}} P_{h, t}^{C H P}+\sum_{g \in \Omega_{n}^{G}} P_{g, t}^{G}+\sum_{w \in \Omega_{n}^{W D}} P_{w, t}^{w e}-\sum_{i \in \Omega_{n}^{E B}} P_{i, t}^{E B}-\sum_{l \in \Omega_{n}^{L D}} P_{l, t}^{l o a d}=\sum_{m \in \Omega_{n}^{N}} B_{n m}\left(\delta_{n, t}-\delta_{m, t}\right), \quad \forall n \in \Phi^{N}, \forall t \in T
$$

Constraints (6)-(7) are generation capacity constraints for CHP units and non-CHP thermal units considering reserve capacity, respectively. Constraints (8)-(9) ensure that each unit provides reserve capacity within its ramping rate.

$$
\begin{gathered}
P_{h, t}^{C H P, \min } \leq P_{h, t}^{C H P}-R_{h, t}^{C H P, D}, P_{h, t}^{C H P}+R_{h, t}^{C H P, U} \leq P_{h, t}^{C H P, \max }, \quad \forall h \in \Phi^{\mathrm{CHP}}, \forall t \in T \\
P_{g, t}^{G, \min } \leq P_{g, t}^{G}-R_{g, t}^{G, D}, \quad P_{g, t}^{G}+R_{g, t}^{G, U} \leq P_{g, t}^{G, \max }, 0 \leq R_{g, t}^{G, U} \leq P R_{g}^{G, u p}, \quad \forall g \in \Phi^{G}, \forall t \in T \\
0 \leq R_{h, t}^{C H P, U} \leq P R_{h}^{C H P, u p}, \quad 0 \leq R_{h, t}^{C H P, D} \leq P R_{h}^{C H P, d o w n}, \quad \forall h \in \Phi^{\mathrm{CHP}}, \forall t \in T \\
0 \leq R_{g, t}^{G, U} \leq P R_{g}^{G, u p}, 0 \leq R_{g, t}^{G, D} \leq P R_{g}^{G, d o w n}, \quad \forall g \in \Phi^{G}, \forall t \in T
\end{gathered}
$$

The output of CHP units and non-CHP thermal units has ramping rate constraints (10)-(11). Constraint (12) limits the electricity consumption of EBs. Constraint (13) is the transmission line limits.

$$
\begin{gathered}
-P R_{h}^{C H P, \text { down }} \Delta t \leq P_{h, t}^{C H P}-P_{h, t-1}^{C H P} \leq P R_{h}^{C H P, u p} \Delta t, \quad \forall h \in \Phi^{\mathrm{CHP}}, \forall t \in T \\
-P R_{g}^{G, d o w n} \Delta t \leq P_{g, t}^{G}-P_{g, t-1}^{G} \leq P R_{g}^{G, u p} \Delta t, \quad \forall g \in \Phi^{G}, \forall t \in T \\
0 \leq P_{i, t}^{E B} \leq P_{i}^{\mathrm{EB}, \max }, \quad \forall i \in \Phi^{E B}, \forall t \in T \\
-f_{n m}^{\max } \leq B_{n m}\left(\delta_{n, t}-\delta_{m, t}\right) \leq f_{n m}^{\max }, \forall n \in \Phi^{N}, \forall t \in T
\end{gathered}
$$

\subsubsection{Day-ahead operation constraints of DHS}

As illustrated in Fig. 1, the DHS consists of heat sources, heat transmission network and end users. The heat transmission network consists of hot water pipelines including supply pipes delivering heat to load nodes, and return pipes carrying mass flow back to heat source nodes.

1) Heat sources 
CHP units and EBs, as the most common heat sources, represent the linkage between the EPS and DHS. CHP units are typically classified into back-pressure and extraction-condensing units [37]. Due to the linear relationship of electric-heating generation, the back-pressure CHP unit is adopted in this paper.

$$
H_{h, t}^{C H P}=\eta_{h}^{C H P} P_{h, t}^{C H P}, \forall h \in \Phi^{\mathrm{CHP}}, \forall t \in T
$$

EBs are considered as electric loads in EPS, which consume electricity to generate heat. The model of the EB is described as bellow.

$$
H_{i, t}^{E B}=\eta_{i}^{E B} P_{i, t}^{E B}, \forall i \in \Phi^{E B}, \forall t \in T
$$

The heat generated from heat sources are used to warm up water or steam and the heat production has correlation with temperatures of supply and return pipelines, as shown in (16).

$$
\sum_{h \in \Omega_{j}^{\mathrm{CHP}}} H_{h, t}^{\mathrm{CHP}}+\sum_{i \in \Omega_{j}^{E B}} H_{i, t}^{E B}=c \cdot m_{j, t}^{H S} \cdot\left(\tau_{n d, t}^{S, H S}-\tau_{n d, t}^{R, H S}\right), \quad \forall j \in \Phi^{\mathrm{HS}}, n d=\Omega_{j}^{H S}, \forall t \in T
$$

2) District heating network

The steady state energy flow model is considered in this paper. The continuity of mass flow rate means that, for each node in the DHN, the mass flows entering the node are equal to the mass flows leaving the node [33], which is depicted as follows.

$$
\begin{aligned}
& \sum_{j \in \Omega_{n d}^{H S}} m_{j, t}^{H S}+\sum_{p \in \Omega_{n d i}^{S, p i p e}} a_{n d, p} m_{p, t}^{S, p i p e}-\sum_{l \in \Omega_{n d}^{H D}} m_{l, t}^{H D}=0, \forall n d \in \Phi^{N D}, \forall t \in T \\
& \sum_{l \in \Omega_{n d}^{H D}} m_{l, t}^{H D}-\sum_{p \in \Omega_{n d}^{R, p l p e}} a_{n d, p} m_{p, t}^{R, p i p e}-\sum_{j \in \Omega_{n d}^{H S}} m_{j, t}^{H S}=0, \forall n d \in \Phi^{N D}, \forall t \in T
\end{aligned}
$$

In the above equations, $a_{n d, p}$ is defined in the following matrix $\boldsymbol{A}$ which is the network matrix that relates the nodes to the pipes in all supply pipelines [38]. The possible values of $a_{n d, p}$ are $+1,-1$ and 0 , which represent the mass flow injects into or leaves from the node $n d$ through pipe $p$, and pipe $p$ does not connect to node $n d$, respectively.

$$
\boldsymbol{A}=\left[\begin{array}{cccc}
a_{1,1} & a_{1,2} & \ldots & a_{1, p} \\
a_{2,1} & a_{2,2} & \ldots & a_{2, p} \\
\vdots & \vdots & \ddots & \vdots \\
a_{n d, 1} & a_{n d, 2} & \ldots & a_{n d, p}
\end{array}\right]
$$

The mass flow passing through the pipeline will cause heat losses. The temperature drops caused by heat losses in the pipeline are given in (20)-(21), which is consistent with the flow direction. Since most district heating systems consist of an underground pipeline network and the temperature variation is relatively small, the ambient temperature is treated as a constant [39].

$$
\begin{aligned}
& \tau_{p, t}^{S, \text { out }}-\tau_{t}^{\mathrm{a}}=\left(\tau_{p, t}^{S, \text { in }}-\tau_{t}^{\mathrm{a}}\right) e^{-k_{p} \pi d_{p} l_{p} / c \cdot m_{p, t}^{S, \text { pipe }}}, \forall p \in \Phi^{S, p i p e}, \forall t \in T \\
& \tau_{p, t}^{R, \text { out }}-\tau_{t}^{\mathrm{a}}=\left(\tau_{p, t}^{R, \text { in }}-\tau_{t}^{\mathrm{a}}\right) e^{-k_{p} \pi d_{p} l_{p} / c \cdot m_{p, t}^{R, \text { pipe }}}, \forall p \in \Phi^{R, p i p e}, \forall t \in T
\end{aligned}
$$

According to the energy conservation law, when the mass flow of pipelines enters a node, the temperature of the mixed fluid is determined by (22)-(23).

$$
\begin{gathered}
\sum_{p \in S_{n d}^{S, p p e}}\left(m_{p, t}^{S, p i p e} \cdot \tau_{p, t}^{S, \text { out }}\right)+\sum_{j \in \Omega_{n d}^{H S}}\left(m_{j, t}^{H S} \cdot \tau_{j, t}^{S, H S}\right)=\tau_{n d, t}^{S, N D}\left(\sum_{p \in \Omega_{n d}^{S, p i p e}} m_{p, t}^{S, p i p e}+\sum_{j \in S_{n d}^{H S}} m_{j, t}^{H S}\right), \forall n d \in \Phi^{N D}, \forall t \in T \\
\sum_{p \in \Omega_{n d}^{R, p i p e}}\left(m_{p, t}^{R, p i p e} \cdot \tau_{p, t}^{R, \text { out }}\right)+\sum_{l \in \Omega_{n d}^{H D}}\left(m_{l, t}^{H D} \cdot \tau_{l, t}^{R, H D}\right)=\tau_{n d, t}^{R, N D}\left(\sum_{p \in \Omega_{n d}^{R, p i p e}} m_{p, t}^{R, p i p e}+\sum_{l \in \Omega_{n d}^{H D}} m_{l, t}^{H D}\right), \forall n d \in \Phi^{N D}, \forall t \in T
\end{gathered}
$$

The temperature of pipelines flowing out of one node are equal to the mixed temperature at this node. 


$$
\tau_{p, t}^{S, i n}=\tau_{n d, t}^{S, N D}, \forall n d \in \Phi^{N D}, \forall p \in \Phi^{S, p i p e}, \forall t \in T
$$

$$
\tau_{p, t}^{R, i n}=\tau_{n d, t}^{R, N D}, \forall n d \in \Phi^{N D}, \forall p \in \Phi^{R, p i p e}, \forall t \in T
$$

3) Heat load

In this paper, a heat load aggregator in the DHS manages all the buildings that connect to the same heat substation, which is represented as one consumption node [25]. Generally, the indoor temperature of customers is centrally controlled and is assumed to be the same. The heat consumption at one node is calculated by (26).

$$
H_{l, t}^{\mathrm{HD}}=c \cdot m_{l, t}^{H D} \cdot\left(\tau_{n d, t}^{S, H D}-\tau_{n d, t}^{R, H D}\right), \quad \forall l \in \Phi^{\mathrm{HD}}, n d=\Omega_{l}^{H D}, \forall t \in T
$$

Due to the large thermal inertia of buildings, the imbalance between heat supply and demand can be temporally accepted in the DHS. The temperature could be maintained for a while when thermal supply changes. Thus, the thermal inertia of building space heating is used, which is reflected by the thermal dynamic process of buildings [39]. When thermal supply is larger than space heating demand, the indoor temperature rises in an acceptable range accordingly, which indicates that the surplus heat can be stored in the building temporarily. To simplify the thermal dynamic process, it is modeled based on the model described in [40], as shown in (27). More detailed description can be found in [41]. The left hand side of (27) is the variation of the indoor air heat energy.

$$
c_{\text {air }} \rho_{\text {air }} V \frac{d T_{l, t}^{\text {in }}}{d t}=H_{l, t}^{\mathrm{HD}}-H_{l, t}^{\mathrm{Build}}, \quad \forall l \in \Phi^{\mathrm{HD}}, \forall t \in T
$$

In order to solve the model, the differential equation shown above is transformed into a discrete difference equation with time interval of one hour $(\mathrm{t})$.

$$
c_{\text {air }} \rho_{\text {air }} V\left(T_{l, t+1}^{i n}-T_{l, t}^{i n}\right)=H_{l, t}^{\mathrm{HD}}-H_{l, t}^{\text {Build }}, \quad \forall l \in \Phi^{\mathrm{HD}}, \forall t \in T
$$

Equation (29) shows the relationship between the heat load used for space heating and the difference between the indoor and outdoor temperatures of buildings.

$$
H_{l, t}^{\text {Build }}=K F\left(T_{l, t}^{i n}-T_{l, t}^{e n v}\right), \quad \forall l \in \Phi^{\mathrm{HD}}, \forall t \in T
$$

Considering human thermal comforts, the indoor temperature of the building could vary in a certain range deviated from the set point.

$$
T_{l}^{i n, \min } \leq T_{l, t}^{i n} \leq T_{l}^{i n, \max }, \quad \forall l \in \Phi^{\mathrm{HD}}, \forall t \in T
$$

For facilitating the discussion in the following section, inequalities (10)-(13) and (30) are transformed into a compact form as in (31), and equalities (14)-(29) are transformed as in constraint (32), since the operation constraints in the second stage are similar as the corresponding constraints in the first stage.

$$
\begin{gathered}
L^{D A}\left(P_{h, t}^{C H P}, P_{g, t}^{G}, P_{i, t}^{E B}, \delta_{n, t}, T_{l, t}^{i n}\right) \leq 0 \\
L E^{D A}\left(H_{h, t}^{\mathrm{CHP}}, H_{i, t}^{E B}, H_{l, t}^{\mathrm{HD}}, \tau_{n d, t}^{S / R, H S}, \tau_{n d, t}^{S / R, H D}, \tau_{p, t}^{S / R, i n}, \tau_{p, t}^{S / R, o u t}, \tau_{n d, t}^{S / R, N D}\right)=0
\end{gathered}
$$

\subsubsection{Real-time regulation constraints of EPS and DHS}

The second-stage problem is formulated to cope with the wind power forecast error in the real-time operation. Based on the uncertainty set and day-ahead schedule from the first stage, the output of thermal units, CHP units and EBs in the IEHS are adjusted adaptively [42]. Constraints (33)-(36) represent that the regulation of generators in real-time operation is restricted within the scheduled reserve capacity in the day-ahead stage. 


$$
0 \leq r_{h, t}^{C H P, U} \leq R_{h, t}^{C H P, U} \quad \forall h \in \Phi^{\mathrm{CHP}}, \forall t \in T
$$

$$
0 \leq r_{h, t}^{C H P, D} \leq R_{h, t}^{C H P, D} \quad \forall h \in \Phi^{\mathrm{CHP}}, \forall t \in T
$$

$$
0 \leq r_{g, t}^{G, U} \leq R_{g, t}^{G, U} \quad \forall g \in \Phi^{G}, \forall t \in T
$$

$$
0 \leq r_{g, t}^{G, D} \leq R_{g, t}^{G, D} \quad \forall g \in \Phi^{G}, \forall t \in T
$$

Constraint (37) represents the power rebalance in the real-time operation. Constraints (38) and (39) denote the limits of the wind spillage and load shedding, respectively.

$$
\begin{aligned}
& \sum_{h \in \Omega_{n}^{C H P}}\left(r_{h, t}^{C H P, U}-r_{h, t}^{C H P, D}\right)+\sum_{g \in \Omega_{n}^{G}}\left(r_{g, t}^{G, U}-r_{g, t}^{G, D}\right)+\sum_{w \in \Omega_{n}^{W D}}\left(\tilde{P}_{w, t}-P_{w, t}^{w e}-W_{w, t}^{s p i l l}\right)-\sum_{i \in \Omega_{n}^{E B}}\left(P_{i, t}^{E B, R T}-P_{i, t}^{E B}\right)+\sum_{l \in \Omega_{n}^{L D}} L_{l, t}^{\text {shed }} \\
= & \sum_{m \in \Omega_{n}^{N}} B_{n m}\left(\delta_{n, t}^{R T}-\delta_{m, t}^{R T}-\delta_{n, t}+\delta_{m, t}\right) \quad \forall n \in \Phi^{N}, \forall t \in T
\end{aligned}
$$

$$
0 \leq W_{w, t}^{\text {spill }} \leq \tilde{P}_{w, t}, \quad \forall w \in \Phi^{W D}, \forall t \in T
$$

$$
0 \leq L_{l, t}^{\text {shed }} \leq P_{l, t}^{\text {load }} \quad \forall l \in \Phi^{E L}, \forall t \in T
$$

Constraints (40) and (41) are the compact form of the operation constraints of the EPS and DHS under uncertainty in the second stage, which are similar to the corresponding day-ahead operation constraints (31) and (32).

$$
\begin{gathered}
L^{R T}\left(P_{h, t}^{C H P, R T}, P_{g, t}^{G, R T}, P_{i, t}^{E B, R T}, \delta_{n, t}^{R T}, T_{l, t}^{i n, R T}\right) \leq 0 \\
L E^{R T}\left(H_{h, t}^{\mathrm{CHP}, R T}, H_{i, t}^{E B, R T}, H_{l, t}^{\mathrm{HD}, R T}, \tau_{n d, t}^{S / R, H S, R T}, \tau_{n d, t}^{S / R, H D, R T}, \tau_{p, t}^{S / R, i n, R T}, \tau_{p, t}^{S / R, o u t, R T}, \tau_{n d, t}^{S / R, N D, R T}\right)=0
\end{gathered}
$$

\subsection{Robust compact formulation}

242 The robust energy and reserve co-optimization scheme described above can be written in a compact matrix form as follows:

$$
\min _{\mathbf{x}} \mathbf{c}^{T} \mathbf{x}+\max _{\mathbf{u} \in \mathbf{U}} \min _{\mathbf{y} \in F(\mathbf{x}, \mathbf{u})} \mathbf{d}^{T} \mathbf{y}
$$

where

$$
\mathbf{A x} \preceq \mathbf{b}, \mathbf{B} \mathbf{x}=\mathbf{a}, \mathbf{x} \in \mathbb{R}_{+}^{\mathrm{n}}
$$

where $\mathbf{x}$ is the first-stage decision vector representing the continuous variables related to the energy and reserve schedule of the EPS and DHS; $\mathbf{y}$ is the second-stage decision vector representing the real-time regulation variables; $\mathbf{u}$ is the uncertain variables of wind power. Constraint (43) represents all constraints in the first stage (5)-(30). Constraint (44) denotes the inequalities in the second stage (33)-(36) and (38)-(40). Constraint (45) refers to (37) and (41). Constraint (46) states that $\mathbf{y}$ are positive variables.

\section{Solution methodology}

$$
\begin{gathered}
F(\mathbf{x}, \mathbf{u})=\{\mathbf{y} \mid \mathbf{C y}+\mathbf{D x} \preceq \mathbf{h} \\
\mathbf{G y}+\mathbf{M u}=\mathbf{g} \\
\left.\mathbf{y} \in \mathbb{R}_{+}^{\mathrm{n}}\right\}
\end{gathered}
$$

The proposed two-stage adaptive robust optimization is solved by the C\&CG algorithm [43], which is a cutting plane based 
method. First, the proposed model is decomposed into a master problem (MP) and a subproblem (SP). Second, the SP is transformed into a mixed-integer linear programming (MILP) with its Karush-Kuhn-Tucker (KKT) conditions. Third, the MP and SP are solved iteratively. In each iteration, the optimal solution of the SP is considered as a significant scenario and new variables and corresponding constraints are added to the MP.

The MP is described as follows, which minimizes the total system operation cost under the worst-case wind realization $\mathbf{u}_{l}{ }^{*}$ obtained from the SP in the previous iteration.

$$
\text { MP: }
$$

$$
\min _{\mathbf{x}, \eta} \mathbf{c}^{T} \mathbf{x}+\eta
$$

s.t.

$$
\mathbf{A x} \preceq \mathbf{b}, \quad \mathbf{B x}=\mathbf{a}
$$$$
\eta \geq \mathbf{d}^{T} \mathbf{y}^{l}, \forall l \in O
$$

$$
\mathbf{C y} \mathbf{y}^{l}+\mathbf{D x} \preceq \mathbf{h}, \forall l \in O
$$

$$
\mathbf{G y}^{l}+\mathbf{M u}_{l}{ }^{*}=\mathbf{g}, \forall l \in O
$$

$$
\mathbf{x} \in \mathbb{R}_{+}^{n}, \mathbf{y}^{l} \in \mathbb{R}_{+}^{n}, \forall l \in O
$$

where $\eta$ is the auxiliary variable, $\mathbf{y}^{l}$ are the new variables generated from the SP and added to the MP, $O$ is the index set for wind uncertainty scenarios $l$, and $\mathbf{u}_{l}{ }^{*}$ is the optimal value obtained from the SP in the last iteration, which is considered as the worst-case realization.

With the optimal result $\mathbf{x}^{*}$ obtained from the MP, the SP below is to identify the worst-case scenario.

SP:

$$
\max _{\mathbf{u} \in \mathbf{U}} \min _{\mathbf{y} \in F\left(\mathbf{x}^{*}, \mathbf{u}\right)} \mathbf{d}^{T} \mathbf{y}
$$

In this paper, the inner min problem of the SP is a linear problem (LP), thus strong duality holds and the KKT conditions are satisfied [44]. Reformulated with the KKT conditions, the max-min SP can be transferred into an equivalent single-level problem which can be solved by commercial solvers.

\section{KKT-SP:}

s.t.

$$
\max _{\mathbf{u} \in \mathbf{U}, \mathbf{y} \in F\left(\mathbf{x}^{*}, \mathbf{u}\right), \lambda, \mu, \mathbf{v}} \mathbf{d}^{T} \mathbf{y}
$$

$$
\lambda_{i} \geq 0, v_{j} \geq 0, \mu \text { is free }
$$

where $\lambda, \boldsymbol{\mu}, \boldsymbol{v}$ are dual variables for constraints (54)-(56), and $i$ and $j$ are the indices of the corresponding constraints. The nonlinear complementary constraints (58)-(59) are linearized with the big-M method [43], and the KKT-SP is converted into a MILP problem. 
The procedure of the $\mathrm{C} \& \mathrm{CG}$ algorithm is conducted iteratively, which is shown in Fig. 2.

(1) Initialization. Set the upper bound as $\infty$ and lower bound as $-\infty$, convergence error $\varepsilon=0.01$, iteration index $l=0$, and $O=\varnothing$;

(2) Solve the MP (47)-(52), and derive the optimal solution $\left(x_{l+1}^{*}, \eta_{l+1}^{*}, y_{l}^{*}, y_{2}^{*}, \ldots, y_{l}^{*}\right)$ and update the lower bound $\mathrm{LB}=c^{T} x_{l+1}^{*}+\eta_{l+1}^{*}$;

(3) Solve the KKT-SP (57)-(61) with the optimal solution $x_{l+1}^{*}$ obtained in step (2), and get the optimal solution $\left(y_{l+1}^{*}, u_{l+1}^{*}\right)$, then update the upper bound $\mathrm{UB}=\left\{\mathrm{UB}, c^{T} x_{l+1}^{*}+d^{T} y_{l+1}^{*}\right\}$;

(4) Convergence check. If UB-LB $\leq \varepsilon$, return $y_{l+1}^{*}$ and terminate. Otherwise, generate new variables $y_{l+1}$ and add corresponding new constraints (49)-(52) to the MP, and update $l=l+1, O=O \cup\{l+1\}$, then go to Step (2).

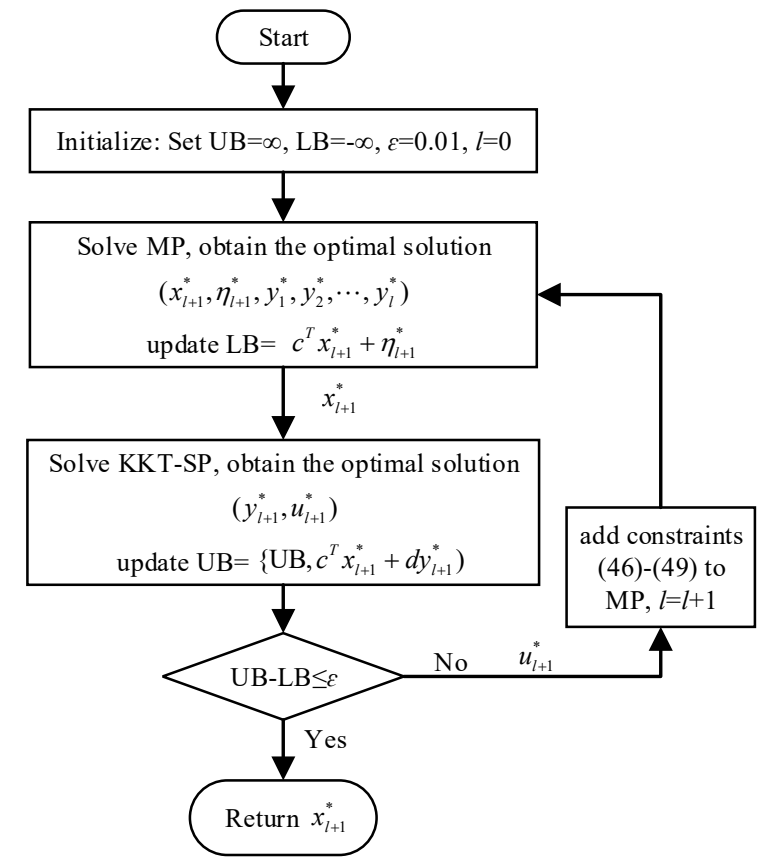

Fig. 2. Solving procedure of $C \& C G$ algorithm

\section{Simulation results}

The topology of the test IEHS is shown in Fig. 3. The system consists of a modified 6-bus system [16] and 4-node DHS [45]. The EPS includes two thermal units (G1 and G2), two wind farms (W1 and W2) and one CHP unit. W1 and W2 with 200 MW and $150 \mathrm{MW}$ installed capacity are located at Bus 2 and Bus 4, respectively. The electricity loads are distributed at Bus 1 and Bus 2. The power to heat ratio of the CHP unit is 1.5 . In the DHS, the mass flow is fixed and the temperature is variable. The supply temperature of the node with heat sources is fixed at $80^{\circ} \mathrm{C}$ to guarantee the quality of heat supply. The temperature in supply pipes varies from $60-80^{\circ} \mathrm{C}$, and the temperature in return pipes depends on the absorption of heat demands. An EB with $12 \mathrm{MW}$ capacity is located at Bus 3 and has a fixed electricity-to-heat ratio of 1 . In this paper, the heat is used for space heating of buildings. The heat demands of each aggregator are assumed to be the same. The standard indoor temperature is set as $20^{\circ} \mathrm{C}$. The minimum and maximum thermal comfort temperature of end users are set to be $18{ }^{\circ} \mathrm{C}$ and $22{ }^{\circ} \mathrm{C}$ [46]. The profiles of hourly electric load and heat load are shown in Fig. 4(a). The predicted and actual deviation of the outputs of two wind farms are shown in Fig. 4(b). The proposed energy and reserve co-optimization scheme was simulated in the General Algebraic Modeling System (GAMS) using the CPLEX. 


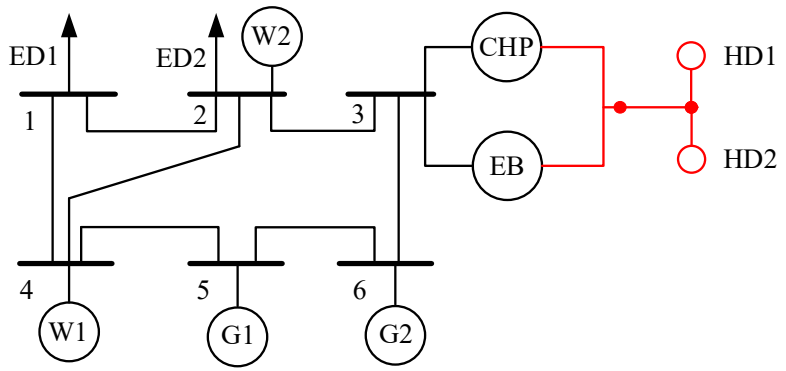

Fig. 3. Test system with 6-bus EPS and 4-node DHS

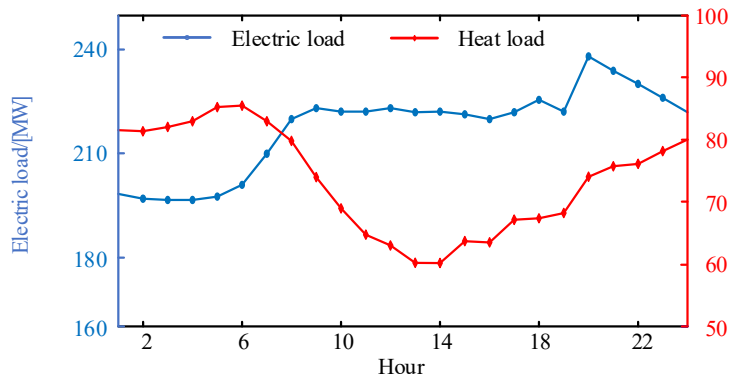

(a)

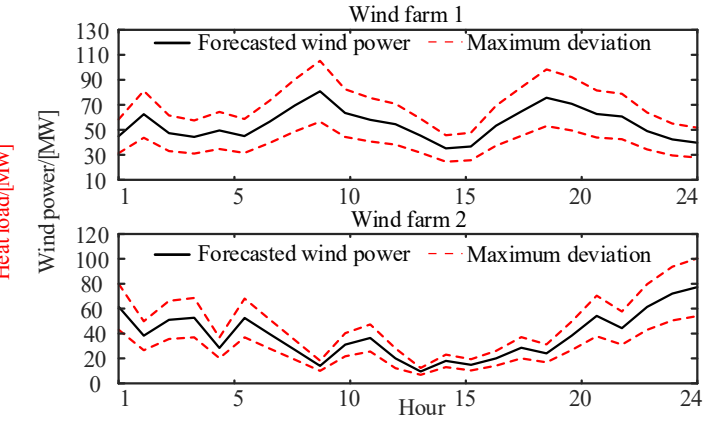

(b)

Fig. 4. Profiles: (a) hourly electric demand and total heat demand and (b) predicted generation and deviation of two wind farms

\subsection{Case study scenarios}

To demonstrate the effectiveness and advantage of the proposed two-stage adaptive robust optimization scheme, a conventional single-stage optimization scheme is used for comparison.

(1) M1: Conventional single-stage energy and reserve optimization model. It is optimized under the pre-defined system reserve requirement without considering the re-dispatch constraints in the real-time stage. CHP units provide full reserve capacity like nonCHP units. The optimized model is formulated as follows [19].

$$
\begin{gathered}
\min \sum_{t \in T}\left(\sum_{h \in \Phi^{\mathrm{CHP}}} C_{h}^{G, C H P} P_{h, t}^{C H P, D A}+C_{h}^{H, C H P} H_{h, t}^{D A}+\sum_{g \in \Phi^{G}} C_{g}^{G} P_{g, t}^{G, D A}+\sum_{h \in \Phi^{\mathrm{CHP}}}\left(C_{h}^{+} R_{h, t}^{U}+C_{h}^{-} R_{h, t}^{D}\right)+\sum_{g \in \Phi^{G}}\left(C_{g}^{+} R_{g, t}^{G, U}+C_{g}^{-} R_{g, t}^{G, D}\right)\right) \\
\sum_{h \in \Phi^{\mathrm{CHP}}} R_{h, t}^{C H P, U}+\sum_{g \in \Phi^{G}} R_{g, t}^{G, U} \geq R U_{t}, \sum_{h \in \Phi^{\mathrm{CHP}}} R_{h, t}^{C H P, D}+\sum_{g \in \Phi^{G}} R_{g, t}^{G, D} \geq R D_{t} \quad \forall t \in T
\end{gathered}
$$

(2) M2: The proposed two-stage robust energy and reserve optimization model. It consists of two stages: day-ahead energy and reserve pre-dispatch and adaptive real-time re-dispatch. The CHP unit provides available reserve capacity that is constrained by EPS and DHS operation constraints in both two stages.

Five case study scenarios are defined. Case 1 is a base schedule without any flexibility in the DHS. EBs and the thermal inertia of buildings, as well as the reserve capacity of CHP units, are not considered in this case. In Cases 2 and 3 , EBs and the reserve capacity of CHP units are considered. In Cases 4 and 5, the thermal inertia of buildings is additionally introduced. Cases 2 and 4 are optimized by M1, while Cases 1, 3 and 5 are optimized by M2. Case details are listed in Table 1.

Table 1 Study cases

\begin{tabular}{ccccc}
\hline Cases & CHP as reserve & EB & Thermal inertia & Optimized method \\
\hline Case 1 & $\times$ & $\times$ & $\times$ & M2 \\
Case 2 & $\sqrt{ }$ & $\sqrt{ }$ & $\times$ & M1 \\
Case 3 & $\sqrt{ }$ & $\sqrt{ }$ & $\times$ & M2 \\
Case 4 & $\sqrt{ }$ & $\sqrt{ }$ & $\sqrt{ }$ & M1
\end{tabular}




\begin{tabular}{lllll} 
Case 5 & $\checkmark$ & $\checkmark$ & $\checkmark$ & M2 \\
\hline
\end{tabular}

\subsection{Results and discussions}

\subsubsection{Feasibility analysis of real-time regulation}

The validity and advantage of the proposed two-stage robust model (M2), as well as its influence on the feasibility of real-time regulation, are firstly analyzed. Fig. 5 shows the comparison results of the real-time power regulation in Cases 2-5. Here, since the cost of wind power spillage is not considered in this paper, neither downward reserve nor downward regulation is scheduled.

From Fig. 5(a), it can be seen that, in Case 2, there is not sufficient available real-time regulation. Since EBs are not scheduled in the day-ahead stage, CHP units cannot increase their outputs in the real-time operation in order to keep heat energy balance. Therefore, when the EPS needs upward regulation, the actual available upward regulation that CHP units can provide is 0. Even though the introduction of buildings thermal inertia enables CHP units to change their outputs to some extent in Case 4, the actual regulation that CHP units can provide is also insufficient, as shown in Fig. 5(c). As such, the electric loads in Cases 2 and 4 have to be shed due to the lack of regulation, which results in the high regulation cost of load shedding in the real-time operation. However, Fig. 5(b) and (d) show that, in Cases 3 and 5, the reserve capacities dispatched in the day-ahead stage are both sufficient for corresponding real-time regulation. The above observations demonstrate that, in the conventional optimization model (M1), the assumption that CHP units have full reserve capacity as thermal units is overoptimistic and this will result in the regulation shortage in the real-time stage.

In summary, the proposed two-stage robust optimization model (M2), considering the real-time operation constraints of the IEHS, can guarantee the feasibility of day-ahead energy and reserve dispatches. Thus, the economic and secure operation of the IEHS considering uncertainty is ensured.

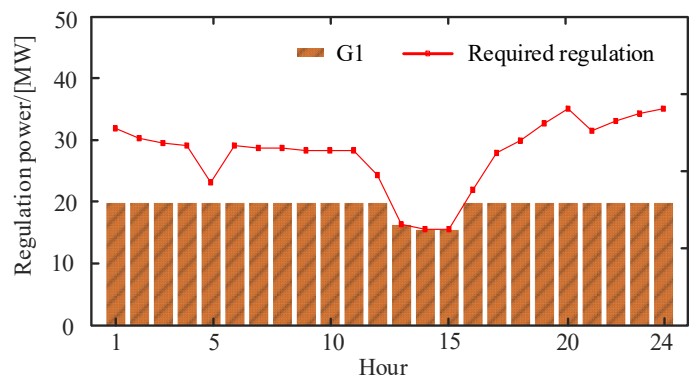

(a)

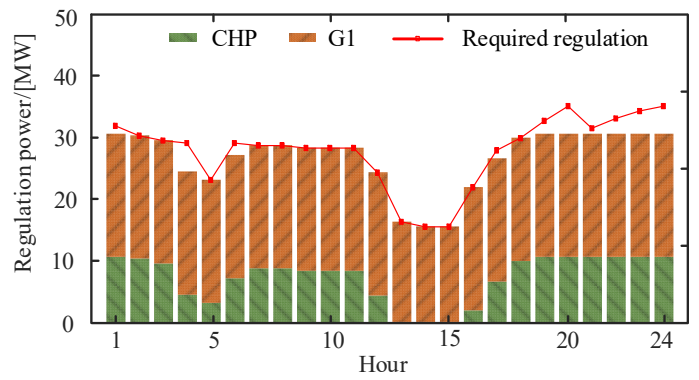

(c)

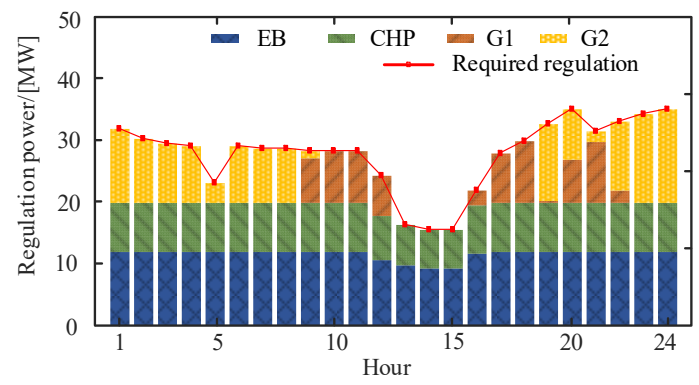

(b)

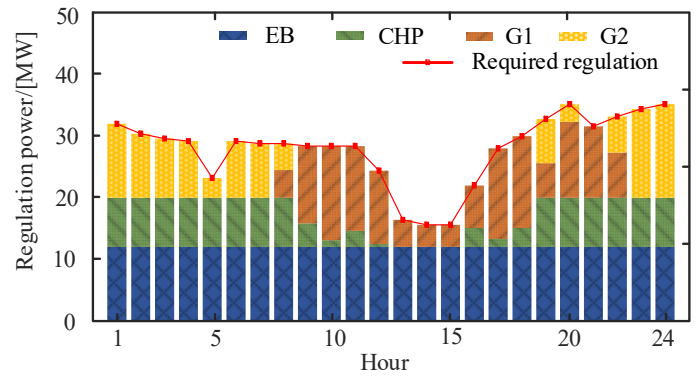

(d)

Fig. 5. Required regulation and actual available regulation of generating units: (a) Case2 (b) Case3 (c) Case4 (d) Case5

\subsubsection{Economic benefits of introducing DHS flexibility}

Cases 1, 3 and 5 with M2 are chosen to evaluate the economic benefits of introducing the DHS flexibility and the additional reserve capacity provided by the DHS. The total system operation costs of the three cases are listed in Table 2, which consists of the costs of energy and reserve dispatches in the day-ahead stage and the regulation cost in the real-time stage. Fig. 6 illustrates 
the optimal dispatches of heat energy and reserve capacity in Cases 1,3 and 5.

Case 1 is the base case without considering EBs, building thermal inertia or the thermal comfort in the IEHS. Thus, there is no flexibility in the DHS. CHP units cannot adjust their outputs and hence non-CHP thermal units are obliged to provide reserve, as shown in Fig. 6(b). According to Table 2, it can be seen that, compared to Case 1, the reserve capacity cost in Case 3 decreases from $\$ 8843.48$ to $\$ 4455.49$. There are two reasons. On the one hand, the EBs in Case 3 enables CHP units to adjust their outputs in the real-time operation and then provide reserve capacity, as shown in Fig. 6(d). Since the reserve price of CHP units is lower than that of thermal unit G2, the reserve capacity cost is reduced. On the other hand, in the two-stage robust optimization, EBs can also serve as reserve to compensate the wind power forecast error. Compared with the reserve capacity provided by CHP units and thermal units, the price of the reserve capacity provided by EBs is relatively cheaper. As such, EBs are scheduled in a priority to meet part of heat demand in the day-ahead stage, which is shown in Fig. 6(c). When the power system needs upward reserve, the EB can lower its output in the real-time operation and hence reduce the consumption of electricity. Since heat supply from EBs is less efficient and more costly than CHP units, the day-ahead energy cost in Case 3 increases a little bit compared with Case 1. Even so, the total system operation cost in Case 3 decreases by $\$ 4513.66$ overall, as shown in Table 2. Therefore, introducing EBs in the IEHS can not only increase the flexibility of CHP units and reduce the operation costs, but also improve the reserve capacity of the IEHS by adopting two-stage robust optimization scheme.

Table 2 Total costs of three cases

\begin{tabular}{cccc}
\hline Costs $(\$)$ & Case 1 & Case 3 & Case 5 \\
\hline Energy cost & 48405.83 & 50599.45 & 50071.50 \\
Reserve capacity cost & 8843.48 & 4455.49 & 4102.90 \\
Regulation cost (worst case) & 9442.19 & 7122.90 & 7012.99 \\
Total cost & 66691.50 & 62177.84 & 61187.39 \\
\hline
\end{tabular}

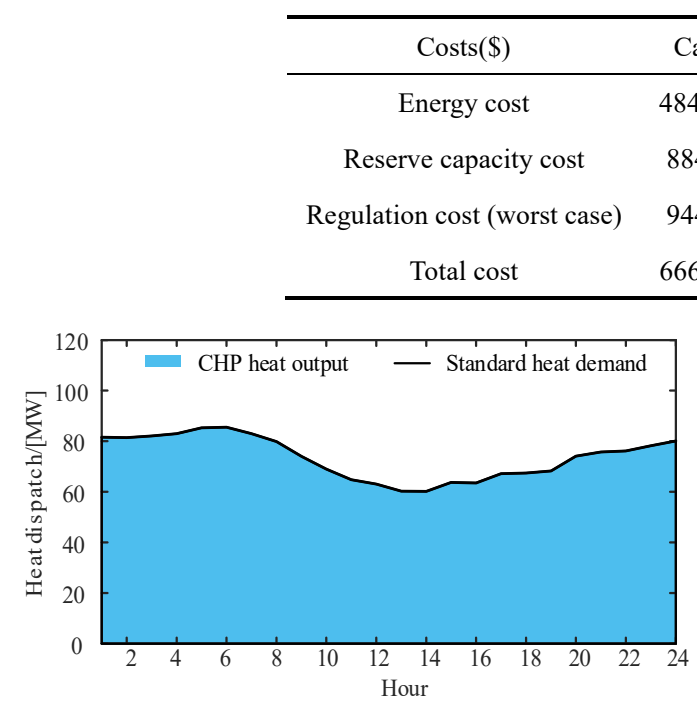

(a) Optimal schedule of heat energy in Case 1

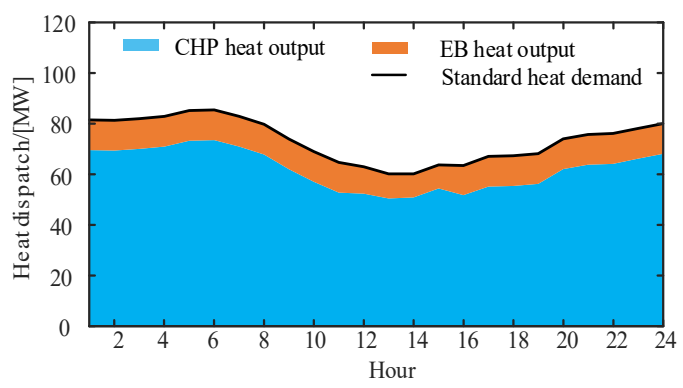

(c) Optimal schedule of heat energy in Case 3

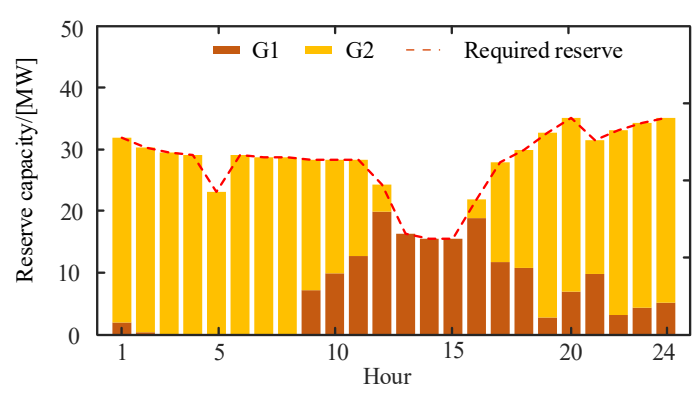

(b) Optimal schedule of reserve capacity in Case 1

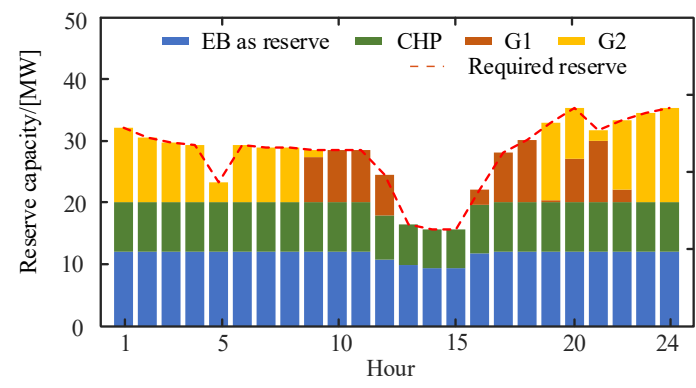

(d) Optimal schedule of reserve capacity in Case 3 


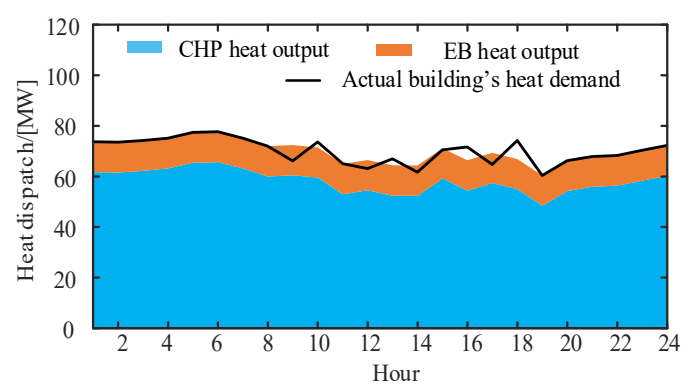

(e) Optimal schedule of heat energy in Case 5

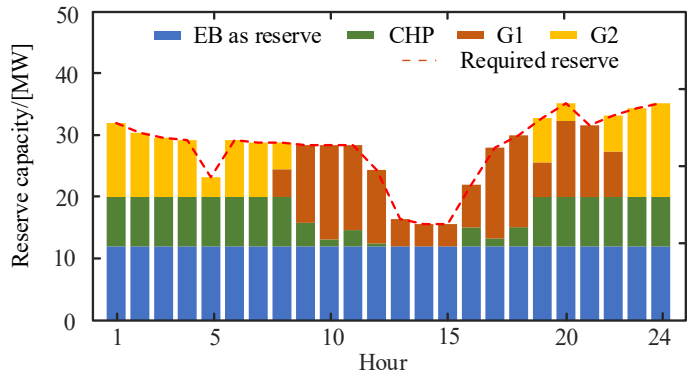

(f) Optimal schedule of reserve capacity in Case 5

Fig. 6. Optimal schedule of heat and reserve capacity in the day-ahead stage

Based on Case 3, the building thermal inertia and thermal comfort are taken into account in Case 5. The indoor temperature can vary in a comfort interval in Case 5. Table 2 shows that, compared with Case 3, the reserve cost in Case 5 decreases by $\$ 352.59$. As shown in Fig. 6(e), the total heat demand of buildings can be temporarily different from the heat supply in Case 5, indicating that the indoor temperature rises and the surplus heat is stored in the building when the heat supply is more than heat demand. Consequently, the flexibility of the CHP is further improved by considering the thermal inertia and thermal comfort. Thus, EBs can be further utilized to provide available reserve capacity and G1 with the cheapest reserve price can provide more reserve in Case 5, which is shown in Fig. 6(f). Therefore, compared with Case 3, the reserve capacity cost in Case 5 is reduced. According to the results, it can be concluded that, with introducing the building thermal inertia into the two-stage robust optimization for the IEHS, EBs can provide more reserve capacity and the total system operation cost can be further reduced.

\subsubsection{Benefits of considering spatial correlations between wind farms}

The wind farm spatial correlation affects the total system costs of energy and reserve schedules. According to [47], the spatial correlation between wind farms is dependent on the wind direction and weather patterns. Generally, the correlation of the outputs of wind farms is high and positive with a short distance, whereas the correlation is negative with an increased distance. Since the interval of wind uncertainty spatial correlation is the input of the proposed robust model, it does not affect the proposed model and solution methodology. Thus, the negative correlation between wind farms is chosen to demonstrate the benefits of considering wind farm spatial correlations. Based on Cases 1, 3 and 5, the proposed robust energy and reserve co-optimization is simulated with and without wind farm spatial correlations. The comparison of corresponding system operation costs in three cases are shown in Table 3. It can be seen that, in all three cases, the reserve capacity cost and total system cost are reduced with the consideration of the spatial correlation of wind farms. This is because the extreme scenarios, when the maximum deviations of two wind outputs happen simultaneously, are not included in the uncertainty set by considering wind farm spatial correlation. Thus, the uncertainty set obtained in this paper covers less area than the typical box uncertainty set and the conservatism of robust solutions is reduced.

Table 3 Total system costs of three cases without and with consideration of wind farm spatial correlation

\begin{tabular}{ccccccc}
\hline \multirow{2}{*}{ Costs $(\$)$} & \multicolumn{3}{c}{ Without correlation } & \multicolumn{3}{c}{ With correlation (95\%) } \\
\cline { 2 - 7 } & Case 1 & Case 3 & Case 5 & Case 1 & Case 3 & Case 5 \\
\hline Energy cost & 48405.83 & 50599.45 & 50071.50 & 48374.36 & 49901.79 & 49480.72 \\
Reserve capacity cost & 8843.48 & 4455.49 & 4102.90 & 8266.95 & 4181.79 & 3882.53 \\
Regulation cost (worst case) & 9442.19 & 7122.90 & 7012.99 & 7317.46 & 5777.69 & 5552.04 \\
Total cost & 66691.50 & 62177.84 & 61187.39 & 63958.77 & 59861.27 & 58915.29 \\
\hline
\end{tabular}

\section{Conclusion}

To cope with the wind power uncertainty, this paper proposes a two-stage adaptive robust energy and reserve co-optimization scheme for the integrated electricity and heat system with utilizing the additional reserve capacity provided by improved flexibility 
from the district heating system and considering the spatial correlation between wind uncertainties. First, electrical boilers and the building thermal inertia are introduced into the district heating system, thus the district heating system can provide additional reserve capacity to the electric power system. In addition, by considering the operation constraints of the electric power system and district heating system in the real-time stage, the optimal schedule of energy and reserve capacity of the integrated electricity and heat system is always guaranteed. The adaptive robust model is reformulated with the Karush-Kuhn-Tucker conditions and is solved by the column-and-constraint generation algorithm. The case study results show that the conservatism of optimized robust solutions is reduced by considering the spatial correlation between wind uncertainties. Compared with the conventional singlestage optimization, the proposed scheme can reduce both reserve capacity cost and the total system operation cost while maintaining secure operation.

\section{Acknowledgements}

414 The PhD student, Jin Tan, is jointly supported by the China Scholarship Council (CSC) (No. 201806270242) and Technical Uni415 versity of Denmark (DTU).

\section{References}

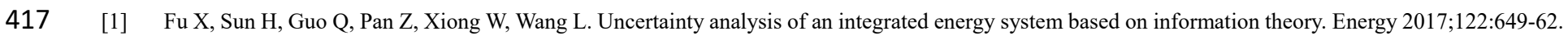

418 [2] Lund H. Large-scale integration of wind power into different energy systems. Energy 2005;30(13):2402-12.

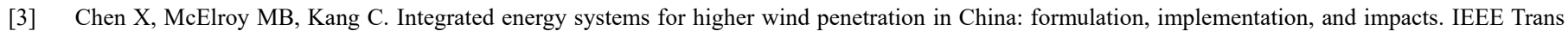
Power Syst 2018;33(2):1309-19.

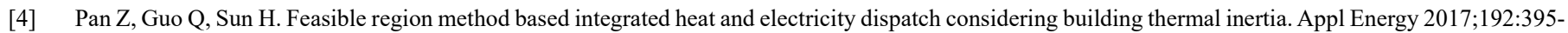
407.

[5] Leśko M, Bujalski W, Futyma K. Operational optimization in district heating systems with the use of thermal energy storage. Energy 2018;165:902-15.

[6] Chen X, Kang C, O'Malley M, Xia Q, Bai J, Liu C, Sun R, Wang W, Li H. Increasing the flexibility of combined heat and power for wind power integration in China: Modeling and implications. IEEE Trans Power Syst 2015;30(4):1848-57.

[7] Zhang N, Lu X, McElroy MB, Nielsen CP, Chen X, Deng Y, Kang C. Reducing curtailment of wind electricity in China by employing electric boilers for heat and pumped hydro for energy storage. Appl Energy 2016;184:987-94.

[8] Li Z, Wu W, Shahidehpour M, Wang J, Zhang B. Combined heat and power dispatch considering pipeline energy storage of district heating network. IEEE Trans on Sustain Energy 2016;7(1):12-22.

[9] Cai H, Ziras C, You S, Li R, Honoré K, Bindner HW. Demand side management in urban district heating networks. Appl Energy 2018;230:506-18.

[10] Yang Y, Wu K, Long H, Gao J, Yan X, Kato T, Suzuoki Y. Integrated electricity and heating demand-side management for wind power integration in China. Energy 2014;78:235-46.

[11] Liu G, Jiang T, Ollis TB, Zhang X, Tomsovic K. Distributed energy management for community microgrids considering network operational constraints and building thermal dynamics. Appl Energy 2019;239:83-95.

[12] Sadamoto T, Chakrabortty A, Ishizaki T, Imura JI. Retrofit Control of Wind-Integrated Power Systems. IEEE Trans Power Syst 2018;33:2804-15.

[13] Yang L, He M, Vittal V, Zhang J. Stochastic Optimization-Based Economic Dispatch and Interruptible Load Management with Increased Wind Penetration. IEEE Trans Smart Grid 2016;7:730-9.

[14] Wang D, Parkinson S, Miao W, Jia H, Crawford C, Djilali N. Hierarchical market integration of responsive loads as spinning reserve. Appl Energy 2013;104:229-38.

[15] Lin C, Wu W, Zhang B, Sun Y. Decentralized solution for combined heat and power dispatch through benders decomposition. IEEE Trans Sustain Energy 2017;8(4):1361-72.

[16] Li Z, Wu W, Wang J, Zhang B, Zheng T. Transmission-constrained unit commitment considering combined electricity and district heating networks. IEEE Trans Sustain Energy 2016;7(2):480-92. 
444 [17] Rinne S, Syri S. The possibilities of combined heat and power production balancing large amounts of wind power in Finland. Energy 2015;82:1034-46.

[18] Sorknæs P, Lund H, Andersen AN, Ritter P. Small-scale CHP as a balancing reserve for wind - the case of participation in the German secondary control reserve. Int J Sustainable Energy Plan Manage 2014;4:31-42.

[19] Wang J, Zhong H, Xia Q, et al. Optimal joint-dispatch of energy and reserve for CCHP-based microgrids. IET Gener Transm Distrib 2017;11(3):785-94.

[20] Caramanis M, Ntakou E, Hogan WW, Chakrabortty A, Schoene J. Co-optimization of power and reserves in dynamic T\&D power markets with nondispatchable renewable generation and distributed energy resources. Proc IEEE 2016;104:807-36.

[21] Li G, Zhang R, Jiang T, Chen H, Bai L, Cui H, Li X. Optimal dispatch strategy for integrated energy systems with CCHP and wind power. Appl Energy 2017;192:408-19.

[22] Wei W, Liu F, Mei S, Hou Y. Robust energy and reserve dispatch under variable renewable generation. IEEE Trans Smart Grid 2015;6(1):369-80.

[23] Zhao B, Conejo AJ, Sioshansi R. Unit commitment under gas-supply uncertainty and gas-price variability. IEEE Trans Power Syst 2017;32:2394-405.

[24] Zugno M, Conejo AJ. A robust optimization approach to energy and reserve dispatch in electricity markets. Eur J Oper Res 2015;247:659-71. Energy 2018.

[26] Arroyo JM, Galiana FD. Energy and Reserve Pricing in Security and Network-Constrained Electricity Markets. IEEE Trans Power Syst 2005;20:634-43.

[27] Liu C, Botterud A, Zhou Z, Du P. Fuzzy energy and reserve co-optimization with high penetration of renewable energy. IEEE Trans Sustain Energy 2017;8:782-91.

[28] Zhang M, Ai X, Fang J, Yao W, Zuo W, Chen Z. A systematic approach for the joint dispatch of energy and reserve incorporating demand response. Appl Energy 2018;230:1279-91.

[29] Bertsimas D, Litvinov E, Sun XA, Zhao J, Zheng T. Adaptive robust optimization for the security constrained unit commitment problem. IEEE Trans Power Syst 2012;28(1):52-63.

[30] Amjady N, Dehghan S, Attarha A, Conejo AJ. Adaptive Robust Network-Constrained AC Unit Commitment. IEEE Trans Power Syst 2017;32:672-83.

[31] Ye H, Li Z. Robust security-constrained unit commitment and dispatch with re-course cost requirement. IEEE Trans Power Syst 2015:1-10.

[32] Pirouti M, Bagdanavicius A, Ekanayake J, Wu J, Jenkins N. Energy consumption and economic analyses of a district heating network. Energy 2013;57:14959.

[33] Cao Y, Wei W, Wu L, Mei S, Shahidehpour M, Li Z. Decentralized Operation of Interdependent Power Distribution Network and District Heating Network: A Market-Driven Approach. IEEE Trans Smart Grid 2018;PP:1.

[34] Chen S, Wei Z, Sun G, Cheung KW, Wang D, Zang H. Adaptive robust day-ahead dispatch for urban energy systems. IEEE Trans Ind Electron 2019;66(2):1379-90.

[35] Cobos NG, Arroyo JM, Alguacil N, Wang J. Robust Energy and Reserve Scheduling Considering Bulk Energy Storage Units and Wind Uncertainty. IEEE Trans Power Syst 2018;33:5206-16.

[36] Hu B, Wu L, Marwali M. On the robust solution to SCUC with load and wind uncertainty correlations. IEEE Trans Power Syst 2014;29:2952-64.

[37] Wang J, You S, Zong Y, Cai H, Træholt C, Dong ZY. Investigation of real-time flexibility of combined heat and power plants in district heating applications. Appl Energy 2019;237:196-209.

[38] Liu X, Wu J, Jenkins N, Bagdanavicius A. Combined analysis of electricity and heat networks. Appl Energy 2016;162:1238-50.

[39] Brahman F, Honarmand M, Jadid S. Optimal electrical and thermal energy management of a residential energy hub, integrating demand response and energy storage system. Energy Build 2015;90:65-75.

[40] Zhang Y, Lin K, Zhang Q, Di H. Ideal thermophysical properties for free-cooling (or heating) buildings with constant thermal physical property material. Energy Build 2006;38(10):1164-70.

[41] Xu X, Zhang Y, Lin K, Di H, Yang R. Modeling and simulation on the thermal performance of shape-stabilized phase change material floor used in passive solar buildings. Energy Build 2005;37(10):1084-91.

[42] Conejo Antonio J, Carrion Miguel, Morales Juan M. Decision making under uncertainty in electricity markets, international series in operations research and management science. 1st ed. New York, NY, U.S.: Springer; 2010

[43] Zeng B, Zhao L. Solving two-stage robust optimization problems using a column-and- constraint generation method. Oper Res Letters 2013;41(5):457/61. 
487 [44] Boyd S, Vandenberghe L. Convex optimization. Cambridge university press; 2004.

488 [45] Turk A, Zeng Q, Wu Q, Hejde A. Optimal operation of integrated electrical, district heating and natural gas system in wind dominated power system. Int. J Smart Grid Clean Energy 2018.

490 [46] Cai H, Ziras C, You S, Li R, Honoré K, Bindner HW. Demand side management in urban district heating networks. Appl Energy 2018;230:506-18.

491 [47] On the Benefits of Distributed Generation of Wind Energy in Europe. [Online]. Available: http://www.drgiebel.de/GGiebel_DistributedWindEnergyInEurope.pdf 